\title{
A Wideband On-Chip Radiator Driven by a Traveling-Wave Photodetector
}

\author{
Craig Ives $^{1^{*}}$, Behrooz Abiri ${ }^{1}$, and Ali Hajimiri ${ }^{1}$ \\ ${ }^{1}$ Department of Electrical Engineering, California Institute of Technology, Pasadena, CA 91125, USA \\ *cives@caltech.edu
}

\begin{abstract}
An integrated broadband Vivaldi antenna driven by an on-chip traveling-wave photodetector is reported. The silicon photonic chip radiates between 21 and $67 \mathrm{GHz}$ with $-65 \mathrm{dBm}$ coupled power at $44 \mathrm{GHz}$. (C) 2019 The Author(s)

OCIS codes: (130.0130) Integrated optics; (130.3120) Integrated optics devices
\end{abstract}

\section{Introduction}

Next-generation telecommunications will rely on increasingly larger bandwidth devices in order to accommodate the exponential growth in data [1]. However, generating broadband and tunable millimeter-wave signals presents a major challenge in pure electronics substrates. Silicon photonics platforms can generate millimeter-wave signals tunable over a broad frequency range with less complexity and smaller die area than their electronic counterparts $[2,3]$. In principle, a photodetector can be used to generate nearly arbitrary frequencies by mixing two lasers together; in practice, photodetectors suffer from RC time constant and transit time bandwidth limitations [4]. The $\mathrm{RC}$ limit can be overcome by designing a distributed photodetector and absorbing the capacitance into a transmission line, while the transit time limit can also be overcome by matching the group velocity of the RF traveling wave to the delay of the optical signal between photodetectors [5]. As a result, properly designed travelingwave photodetectors (TWPD's) are primarily limited by the loss of the transmission line, rather than group velocity walk-off. This work pushes against the limits of these bandwidth constraints in a demonstration of a wideband Vivaldi antenna driven by a traveling-wave photodetector, all fully integrated in a silicon photonics process.

\section{Traveling-wave photodetector}

The photodetector used in this design is dual fed with germanium only on top of the waveguide (Fig. 1a). This reduction in germanium area allows higher optical powers to be absorbed, and feeding from both ends of the photodetector increases efficiency [6]. For an individual TWPD, the photodetectors are placed in parallel as part of the transmission line between the GSG pads [7], in which one side is a termination (L) and the other side is the receiver (S) (see Fig. 1c, bottom). The input optical signal is split and fed to the different photodiodes through progressively increasing optical delays, which are realized as meandering optical waveguides that match the propagation velocity of the electrical transmission line. The devices were fabricated in the IME silicon photonic process, with a waveguide rib height of $220 \mathrm{~nm}$ and a slab height of $90 \mathrm{~nm}$.

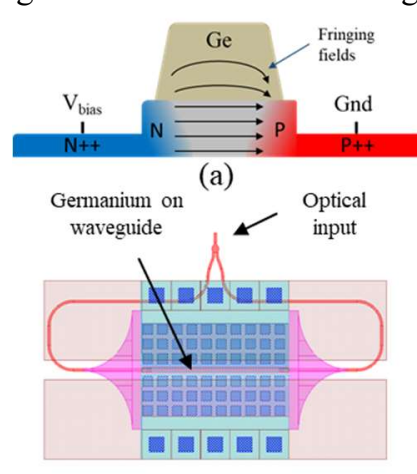

(b)

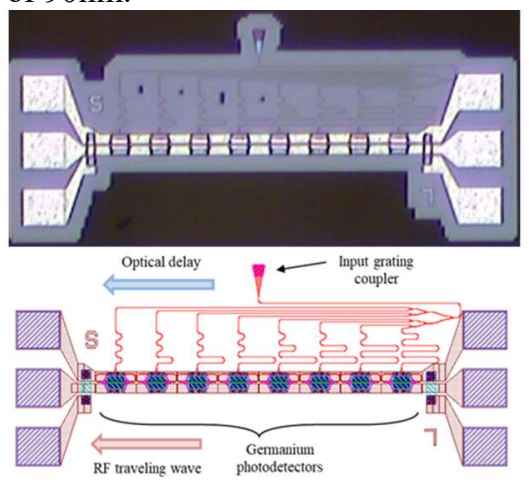

(c)

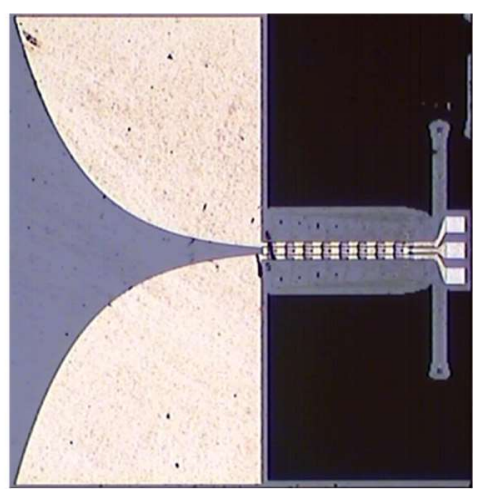

(d)

Figure 1: a) Cross section of the photodiode. b) Layout of the individual germanium photodiode. c) Layout and micrograph of a full TWPD. c) Micrograph of the radiator chip, which measures $1.8 \times 1.8 \mathrm{~mm}$.

\section{Vivaldi antenna}

The radiator is a Vivaldi antenna fed by a slot waveguide, consisting of two parallel TWPD's that share a common electrode. The pads for a SGS probe serve as a termination and as a means to align the two fibers. Simulations predict a peak gain of $3.5 \mathrm{dBi}$ at $150 \mathrm{GHz}$, and a gain of $0.8 \mathrm{dBi}$ at $50 \mathrm{GHz}$. 


\section{Measurement results}

A $1.14 \mathrm{~mm}$ TWPD, whose frequency response is shown in Fig. 2a, exhibited a $3 \mathrm{~dB}$ bandwidth of $32 \mathrm{GHz}$, limited by loss in the transmission line, while the radiator exhibited $-65 \mathrm{dBm}$ of coupled power at $44 \mathrm{GHz}$, as shown in Fig. 3 . The power drops closer to $20 \mathrm{GHz}$ due to the lower radiation efficiency of the Vivaldi, and drops at higher frequencies due to the loss in the TWPD.

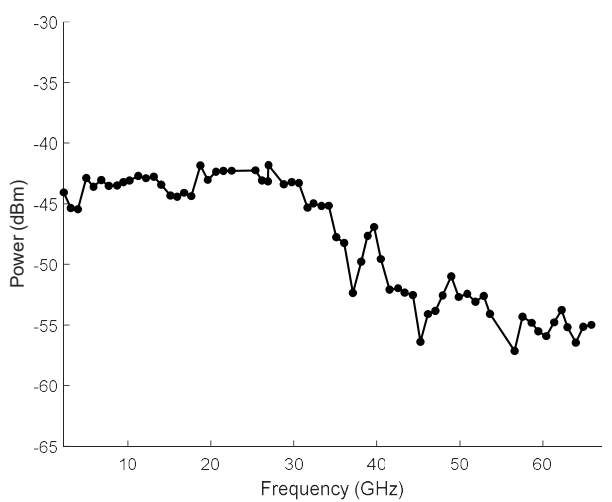

(a)

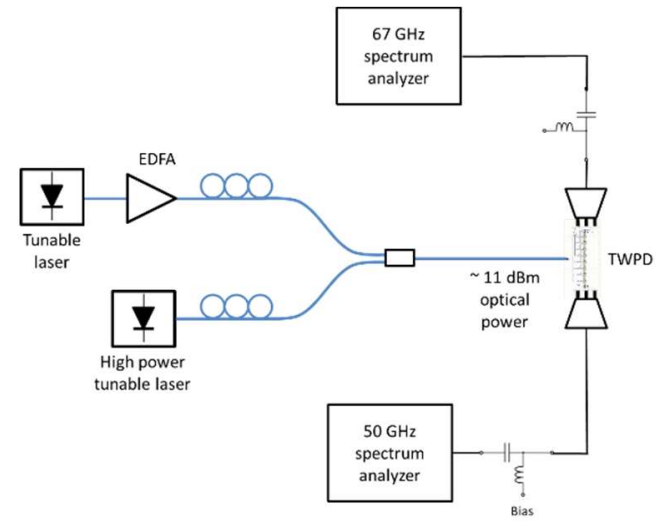

(b)

Figure 2: a) Frequency response of a 1.14mm long TWPD with a $3 \mathrm{~dB}$ bandwidth of $32 \mathrm{GHz}$. b) The experimental setup for measuring the TWPD.

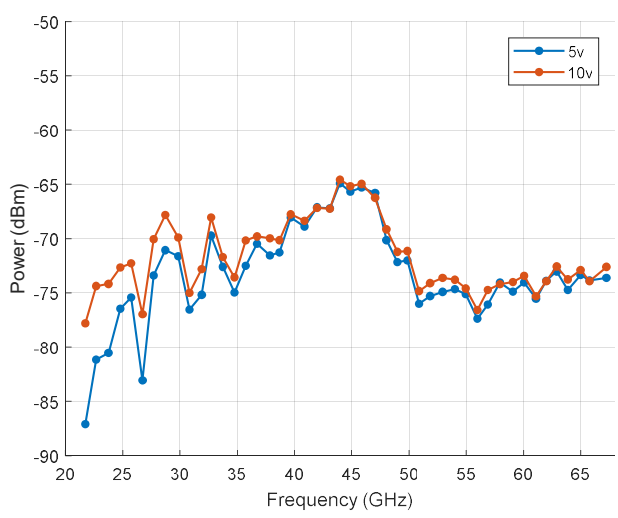

(a)

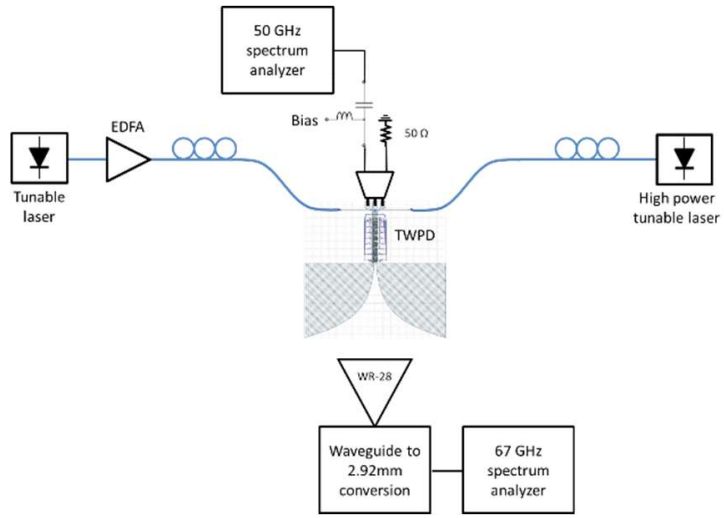

(b)

Figure 3: a) The Vivaldi's coupled power when reverse biased at $5 \mathrm{~V}$ and $10 \mathrm{~V}$. Each data point is the average of ten measurements, with steps of $1 \mathrm{GHz}$. b) Experimental setup for measuring the radiated power. For the 40-67 GHz band, the WR-28 horn is replaced with a WR-15 horn.

\section{References}

[1] J. G. Andrews, S. Buzzi, W. Choi, S. V. Hanly, A. Lozano, A. C. K. Soong and J. C. Zhang, "What Will 5G Be?," IEEE Journal on Selected Areas in Communications, vol. 32, no. 6, pp. 1065-1082, 2014.

[2] T. Nagatsuma, G. Ducournau and C. C. Renaud, "Advances in terahertz communications accelerated by photonics," Nature Photonics, vol. 10, pp. 371-379, 2016.

[3] J. Moody, K. Sun, Q. Li, A. Beling and S. M. Bowers, "A Vivaldi Antenna Based W-band MUTC Photodiode Driven Radiator," in IEEE International Topical Meeting on Microwave Photonics, Long Beach, 2016.

[4] J. E. Bowers and C. A. Burrus, "Ultrawide-Band Long-Wavelength p-i-n Photodetectors," Journal of Lightwave Technology, vol. 5, no. 10, pp. 1339-1350, 1987.

[5] K. S. Giboney, M. J. W. Rodwell and J. E. Bowers, "Traveling-Wave Photodetector Theory," IEEE Transactions on Microwave Theory and Techniques, vol. 45, no. 8, pp. 1310-1319, 1997.

[6] C.-M. Chang, J. H. Sinsky, P. Dong, G. de Valicourt and Y.-K. Chen, "High-power dual-fed traveling wave photodetector circuits in silicon photonics," Optics Express, vol. 23, no. 17, 2015.

[7] X. Luo, J. Song, X. Tu, Q. Fang, L. Jia, Y. Huang, T.-Y. Liow, M. Yu and G.-Q. Lo, "Silicon-based traveling-wave photodetector array (SiTWPDA) with parallel optical feeding," Optics Express, vol. 22, no. 17, 2014. 